\title{
How the Knowledge Economy is Transforming the City from the Inside
}

MAR SANTAMARIA-VARAS

$300.000 \mathrm{Km} / \mathrm{s}$

The knowledge economy is transforming our cities. The return of large companies' headquarters to city centers and the proliferation of innovation districts in metropolises around the world represent a paradigm change. Today, scientific literature focuses on the metrics of innovative activity from the point of view of human capital and creative workers, global economic indicators, company characteristics, scientific production or urban implantation. However, there is a lack of research demonstrating that the knowledge economy has its own urban form that depends on the characteristics of the site - innovation activities are more likely to emerge in specific urban conditions. This paper aims to frame the emergence of innovative urban economy in an international context and describe a case study in the Barcelona metropolitan area, in line with the aforementioned hypothesis. Place matters in the leverage of innovation.

\section{INTRODUCTION}

According to recent scientific literature, the geography of innovation is changing. Large companies are returning to cities. International giants such as Google have been progressively diversifying their R\&D\&I headquarters located in Mountain View and moving them to New York, London and Pittsburgh, which represent new urban ecosystems focused on technology with state-of-the-art research institutions and a supply of qualified workers.

Over the past 50 years, the urban model of innovation has been based on the Silicon Valley model: a suburban corridor of corporate campus partnerships (to protect the business secret), accessible only by car and in which the quality of life and the integration of housing, work and residency were not a priority. In contrast to this earlier model, and as Katz \& Wagner (2014) have confirmed, it would appear that an alternative paradigm is emerging: innovation districts. These are compact enclaves in central areas of urban cores, rich in different facilities and services. They feature a good mix of companies, recently created startups and educational institutions - all connected via public transportation, powered by clean energy and conjoined through optimal infrastructure for the transfer of information.

As posited by the authors, cities such as New York, London, Eindhoven, Medellín, Montreal, Seoul, Berlin, Boston and Chicago boast consolidated or developing innovation districts.

\author{
PABLO MARTINEZ-DIEZ \\ $300.00 \mathrm{Km} / \mathrm{s}$
}

These have been implemented either by anchor institutions (typically private or public universities), through the urban renewal of unused areas with the goal of promoting the establishment of innovation initiatives or through the renovation of existing research parks in order to integrate them into the urban area and territorial structure.

While it is possible to observe a clear trend in the emergence of innovation districts in cities and metropolises of various types, it can take up to 30 years for the urban and economic success resulting from the implantation of activities related to innovation to become visible. In this sense, it is crucial to measure how this phenomenon comes about both on an urban and territorial level, what variables influence the positive implantation of innovation activities in cities and how the social and economic benefits of such activities are transferred.

Prior studies have, in fact, laid our strategies to measure this impact. On the one hand, Guzman and Stern (2015) argue that company characteristics (name, legal structure, belonging to a partnership, control over intellectual property, etc.) are key factors in its success and completely disregard its location as a determining factor. On the other hand, several studies base cities' potential for innovation on their capacity to produce scientific research (Elsevier, 2015). This is analyzed by measuring indicators such as the number of universities and research centers and the corresponding scientific production.

Lastly, a large number of studies have constructed indicators that measure innovation through demographic and macroeconomic analysis: from Richard Florida's hypothesis, which emphasizes the crucial role of human capital in urban economy and the capacity of urban regions to attract talent, to research carried out by Van der Beerg and Boix, which takes into account the base that makes knowledge production possible, be it through the study of global indicators or through data related to occupation (number of workers, economic sectors, qualification) and companies (livestock, production, exportation, etc.).

In contrast to the aforementioned studies, the research presented in this article, titled "Geographies of Innovation in the Barcelona Metropolitan Area" stems from the hypothesis that innovation has a unique urban form that depends on the characteristics of each site, or rather, innovation initiatives are more likely to emerge in specific urban conditions. 

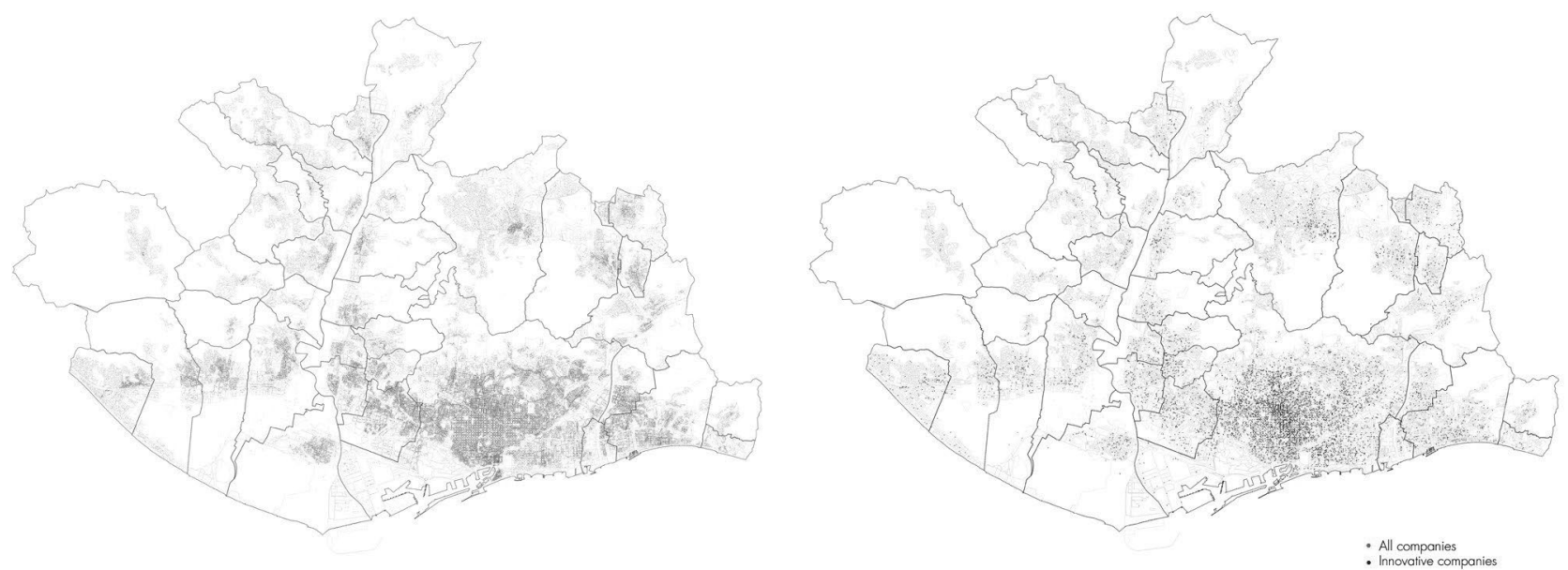

Figure 1: Jewett Arts Center from southwest, ca. 1958, Wellesley College Archives.

Thus, factors such as building density, rent price, close urban services, quickly accessible public transportation and symbolic representativeness, among others, may be key to determining the clustering of activities in certain places within the territory, given different spatial patterns.

Based on the understanding that the conditions of urban fabric influence the generation of different economic and spatial models, and using the case study of the Barcelona metropolitan area, this study aims to asses the impact of innovation initiatives in the territory. The objective is to determine what these initiatives are and what synergies are generated between them, as well as to describe the urban fabric of Barcelona and its 35 surrounding municipalities in function of their potential to host this kind of activity. How can we create innovation fabric and strengthen that which already exists? What variables determine the emergence of this kind of activity and how can we act on these parameters to make them more efficient?

\section{THE BARCELONA METROPOLITAN AREA:}

\section{PRODUCTION AND ECONOMIC CONTEXT}

Throughout history, the Barcelona metropolitan area has been known for welcoming new ideas and promoting innovation. The symbiosis between production capacity and urban development represents an important dynamic within the territory. However, over the past 40 years, the industrial economy has been complemented by an economy based on production, individual and knowledge services.

Barcelona is currently a knowledge city, with a solid knowledge base and a steady and well-maintained growth trajectory in the structure of knowledge.
In particular, the city is a worldwide reference for new technology (Internet and mobile services and software, e-commerce, Big Data, tourism). According to the CITIE Index, Barcelona presents favorable conditions regarding the cost and quality of life, the presence of local and international talent, excellent connectivity and a strategic position, affordable office spaces, access to venture capital, the existence of company incubators, international events and a network of specialized universities and cases of business success.

Likewise, the territory surrounding Barcelona presents highly favorable conditions per se for becoming a knowledge metropolitan, thus strengthening the individual synergies originating in Barcelona at the metropolitan level . A total of $60 \%$ of workers in knowledge-intense activities and $68 \%$ of workers in creative activities in Catalonia are located in the metropolitan area. With 961 patents registered between 2005 and 2012, many companies have located their headquarters in the metropolitan area.

In recent years, local and regional authorities have implemented active policies designed to promote the development of facilities based on value-added knowledge, such as universities, while public and private consortia have endorsed the development of new technological parks and support services for entrepreneurial initiatives that complement a consolidated industrial fabric.

Likewise, the urban planning carried out over the past 40 years has prepared the land for the development of economic activity. The productive backbone of the metropolitan area is formed by industrials uses, offices and commerce, which have been developed along large road and rail networks, the main territorial and civic axes and on the ground floor of urban districts, respectively. 


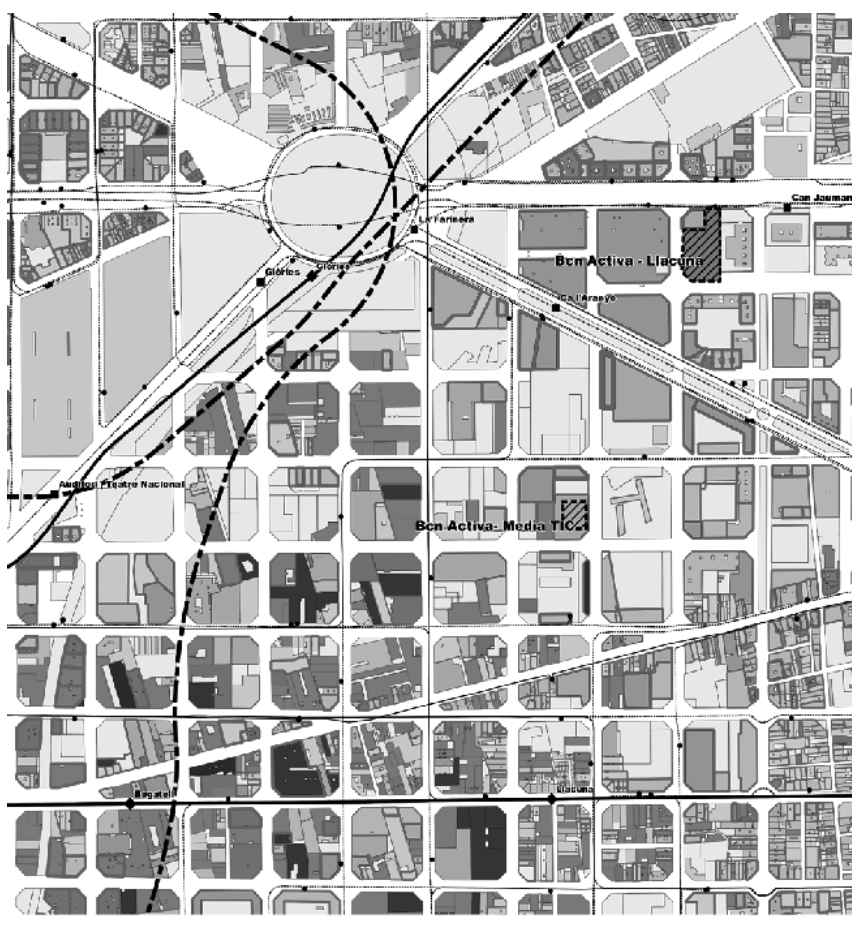

Figure 2: Variables of the urban fabric.

This distribution of land is reflected by an economic fabric composed of 315,000 companies scattered throughout the territory, around $19,000(6 \%)$ of which are dedicated to innovative economic sectors according to the Spanish Ministry of Economy's activity classification . Bearing in mind such criteria, the innovation panorama in the Barcelona metropolitan area can be considered positive. However, the method employed does not offer a qualitative characterization of the most relevant agents and initiatives, as there is a significant bias when only considering economic sectors tied to technology.

It's important to consider that innovation is a changing phenomenon that must be measured using both statistical data and dynamic data that make it possible to establish a framework for comparison of different cities (for example, search platforms for specialized jobs).

For this reason, a geolocation database with nearly 2,250 innovation initiatives in the Barcelona metropolitan areas was created using existing data sources, such as official organizations (Barcelona Metropolitan Area agency, the Agency for Business Competitiveness of the Government of Catalonia, the Spanish Ministry of Economy and Business), existing inventories (Mapa de la Recerca, Barcelona Startup Map, universities) and specific platforms (AngelList). Prior to carrying out this study, there were no existing databases that offered a global view of innovation initiatives in the Barcelona metropolitan area.

\section{THE INNOVATION ECOSYSTEM IN THE BARCELONA METROPOLITAN AREA}

In the field of innovation and new technologies, emerging innovation businesses are understood to be companies that, despite their youth and lack of resources, achieve market results and reach higher structural levels as they are fueled by other investors or absorbed in the future by already consolidated companies.

Based on this definition, innovation companies require an innovation ecosystem in order to flourish. The term is employed to describe the diverse set of participants and resources that contribute to and are necessary for the generation of innovation. That includes companies, investors, researchers, universities and venture capital, as well as business development services and other technical service providers, such as designers, manufacturers, contractors and professional training and development providers. It's worth noting that innovation is considered successful when the knowledge accumulated at research centers is transferred to small, specialized companies that then feed knowledge to large companies that are able to bring innovation to the general public.

In the case of the BMA, the innovation system comprises 2,244 initiatives divided into four large groups of agents:

- 349 research entities, grouped into research centers, research facilities and universities/university campuses;

- 700 success stories, of small, medium and large companies that are relevant due to a variety of factors (attraction capacity, innovation model, etc.);

- 297 support infrastructures, including services (either related to assessment or space) offered to companies by different local and supramunicipal administrations and private institutions, and other agents that promote the innovation ecosystem by accelerating companies and their financing;

- 898 emerging innovation companies, which, as previously mentioned, constitute the base and driver of the innovation ecosystem.

All of these agents have a specific spatial distribution within the territory. For example, the majority of research entities take on the form of campus partnerships and are found only in some municipalities. By contrast, startups present greater variability: from sectors that seek out centrality and services available in urban centers, to economic activities that prefer industrial fields with storage space available and rapid accessibility to road networks, and sectors located close to research centers where basic research is produced.

Given such variety, an analysis was carried out on the territorial level and a study of exemplary cases was conducted on an urban level. The first consisted of isolating the fields with 


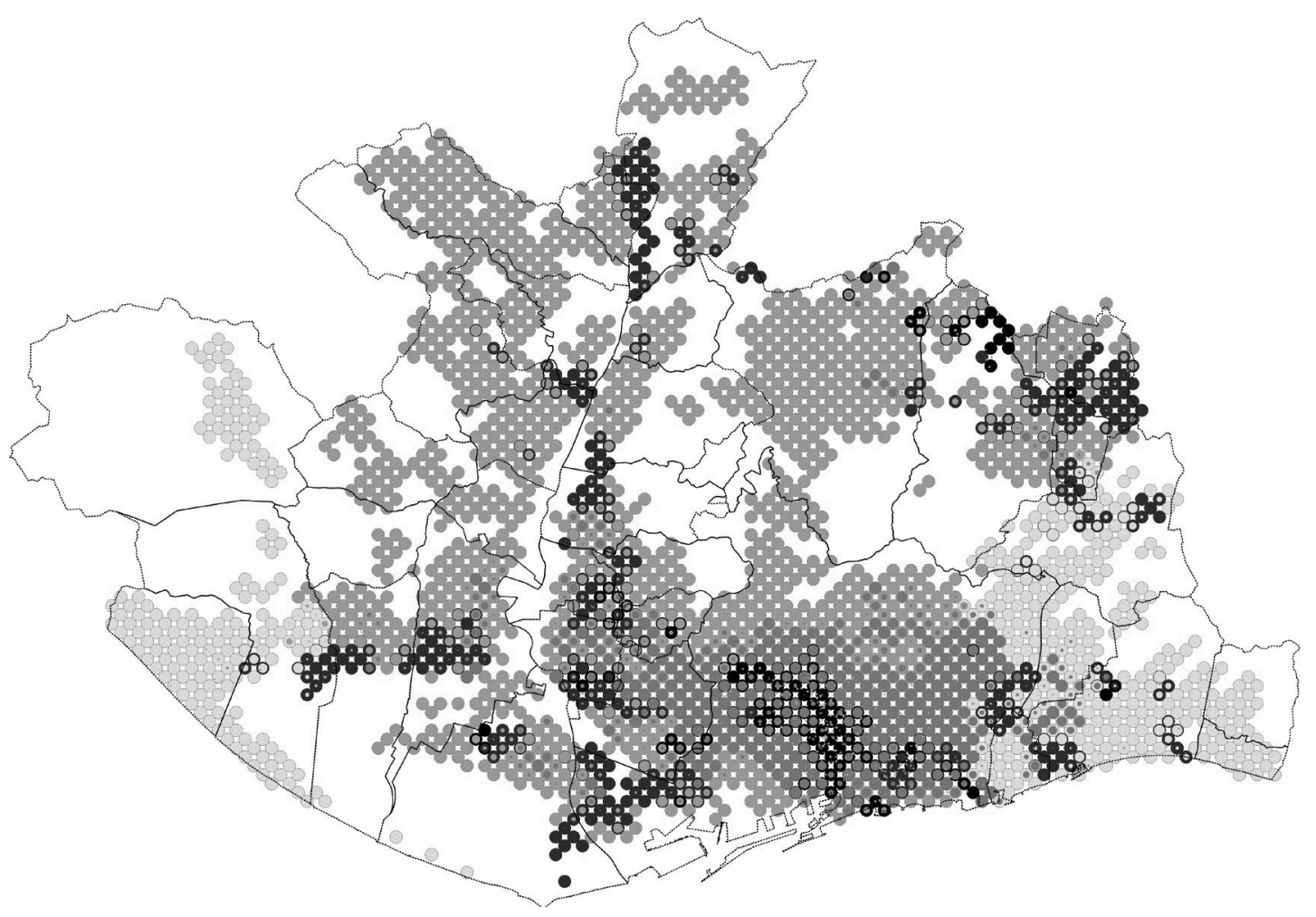

Figure 3: Definition of different urban fabric groups. Geografies de l'innovació a l'AMB

the greatest concentration of emerging innovation initiatives (main research focus) and assessing the weight of various morphological, economic and connectivity parameters, with the main objective of determining whether or not there exist strong correlations between these variables and the appearance of emerging innovation initiatives.

In addition to obtaining detailed verification of the conclusions drawn regarding the urban fabric on the territorial level, the second study aimed to examine the interrelationship between the participating agents of six specific ecosystems: an innovation district (Barcelona 22@), a city lab, two university campuses, a technological business park and an industrial park (the new headquarters of the Amazon logistics center in Spain).

In both cases, the number and complexity of variables to be analyzed (about 75) exceeded the synthesis capabilities of the mapping tools, thus making it necessary to employ statistical analysis methodologies and data mining techniques.

The first phase of the strategy employed consisted of constructing a data model that learned algorithms and thus allowed the identification of the weight of each variable in a fixed number of emerging innovation companies in a specific point in the territory. In the second phase, the data model was capable of predicting where else this phenomenon might occur.
To this end, two databases were used in parallel: the innovation initiatives database (2,244 entries) for algorithm training and the cadastral registry database $(197,000)$ for the prediction phase. Both databases contain variables related to innovation initiatives (for example, the type of agent or sector of the activity); urban morphology (the Cadaster); the economic fabric; and accessibility to different transportation systems.

The results obtained in the first phase of calculation confirm that the parameters that influence the presence of emerging innovation companies are related to building density, the existence of land dedicated to production activities (commerce, offices and industry) and good connectivity to high-frequency transportation systems.

These are all urban parameters that have a decisive impact on the emergence of innovation and yet, at the same time, are susceptible to modification through specific public policies in areas where a deficit or a potential interest is detected.

For example, plans could be developed to generate or transform land for knowledge activities with a greater or lesser occupation density and a mix of suitable uses that permit the implantation of emerging innovation companies with disparate needs. Moreover, and as would be the case for the Barcelona metropolitan area, public policies could also facilitate improvements to internal connections between 


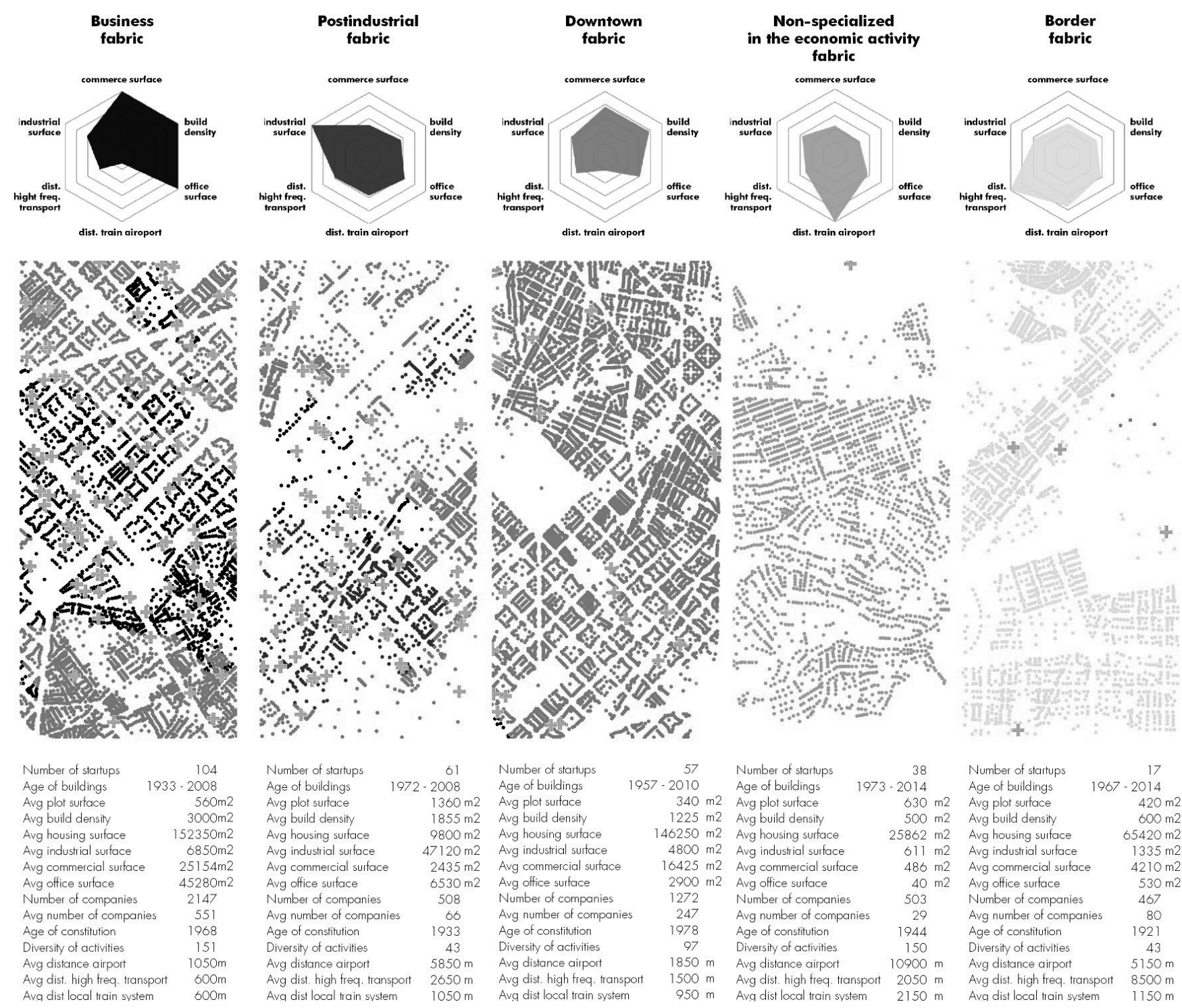

Figure 4: Characteristics of different urban fabric groups.

transportation systems, the connection between metropolitan cities and international connections. This is a crucial variable for converting particularly interesting urban fabric into innovation fabric.

Likewise, the location and planning of strategic pieces would need to be emphasized within the field of urbanism.

For instance, universities, research centers and other facilities linked to innovation must be located in areas with activated fabric in order for an urban return to occur and for other innovation initiatives to be set up in surrounding areas. Similarly, the creation of support facilities and infrastructure for companies (accelerators, business incubators, publicly promoted co-working spaces, etc.) must take place in strategic locations so they can provide real support to their target agents and become facilitators of innovation fabric.

\section{THE TRANSFORMATION POTENTIAL OF INNOVATION FABRIC}

This research proposes an analysis and action method that can be applied to other urban contexts, as well as a method for creating data, most of which are also available in other international metropolises.

However, the research also aims to predict whether or not there are territories with favorable conditions for hosting innovation.

That is why, in the case of the Barcelona metropolitan area, a classification of the urban fabric was carried out according to its potential for hosting innovation initiatives. This classification was based on a clustering strategy and used the previously obtained input variables. Five groups were established: business fabric, post-industrial fabric, center fabric, non-production fabric and surrounding fabric. 
It's worth commenting that this classification is the result of input parameters that are characteristic of a compact city. The application of this methodology in other urban contexts would result in different predictions with necessarily different morphological characteristics. Nevertheless, these would allow for the comparison of very different urban realities. This brings us to the last part of this paper: this research is significant in a global context of growing urbanization, in which new phenomena are transforming cities from the inside.

Thus, the knowledge economy is generating novel economic forms: new urban morphologies, which in many cases acquire dynamics and spatial logics that diverge from traditional ones. This disruption is generating impacts that urban planning is not yet able to identify, trace or mold using traditional land classification strategies and other methodologies.

This new economy is one hundred percent urban. It does not occur in the outskirts and peripheries. It does not result in isolated, monofunctional parks outside of the city, where noise, pollution, and the impact of logistics are invisible. These activities infiltrate city centers. They offer workers the value of urban life with its associated services and facilities. It is a silent economy, with a low environmental impact on its immediate surroundings. It requires places that meet both functional and symbolic requirements that help attract talent, create a corporate identity and transform physical space into a symbolic place - which teleworking is not able to offer.

These new activities are discreetly creating their own spaces within our cities. They sometimes occupy ground floors once used as parking lots or recycle stores that e-commerce has freed up, as it does not require proximity to customers. Others are located on land intended for residential use, thus taking advantage of their ambiguous classification, which allows them to mix with inside dwellings or replace them. They also appear in an organized and planned manner, as part of a strategic economic promotion exercise.

The incorporation of these new economic activities into urban fabric is radically transforming urban environments. They not only balance the proportion of land uses but they also generate new demands for services that either complement and enrich existing ones or generate new exclusive facilities that could result in the segregation of different populations (workers and residents) that share a territory in interlaced temporal segments.

Although urban planning itself cannot guarantee the economic success of companies, it is a crucial factor in the leverage of innovation activities and, more importantly, in establishing synergies between the different activities located in the urban environment. With its land plots and density, the city generates a porosity that facilitates the exchange and transfer of knowledge between elements.
In a context in which spatial relationships have lost their functional value - due to teleworking and logistics networks-, places now embody symbolic and decisive value when it comes to influencing the location of new economic activities. In a globalized world, where novel paradigms of transportation and commerce free up large land plots in cities, the return of the economy from industrial peripheries to urban centers is an opportunity to envision more livable urban milieus.

\section{ENDNOTES}

1. B. Katz and J. Wagner, "The Rise of Innovation Districts: A New Geography of Innovation in America," report, Metropolitan Policy Program at the Brookings Innovation in America,
Institution, May 2014.

2. B. Katz and J. Wagner, "The Rise of Innovation Districts," Harvard Business Review, November 12, 2014.

3. J. Guzman and S. Stern, "Where is Silicon Valley?" Science 6 (2015).

4. “Mapping Research and Innovation. Understanding Amsterdam's Competitive Advantage," report, the Urban Innovation Network and Elsevier, 2015.

5. R. Florida, "The Economic Geography of Talent," Annals of the Association of American Geographers 92, no. 4 (December 2002): 743-755.

6. Barcelona Metropolitan Area Innovation mapping is based on experience gained through previous research on the innovation ecosystem of Barcelona gained through previous research on the innovation
carried out by our team, see: http://goo.gl/UWGY7V.

7. According to the authors, knowledge cities are classified as such based on the existence of a knowledge base, type of economic base, quality of life, accessibility, urban diversity, urban dimension and social equality. See L. Van Den Berg et al "European cities in the Knowledge Economy," in Euricur Report R2004-1 (Rotterdam, 2004).

8. CITIE is a partnership between Nesta, Accenture and the Future Cities Catapult. Indicators are categorized into three types: openness, infrastructure supply and the city's innovation in performing its activity.

9. J. Trullén, L'economia de Barcelona cap a l'economia del coneixement: diagnosi económica i territorial de Barcelona, Barcelona City Council, GTP (2001)

10. R. Boix, Barcelona Ciutat del Coneixement: Economia del Coneixement, Tecnologies de la Informació i la Comunicació i Noves Estratègies Urbanes, Barcelona City Council, GTP (2005).

11. According to data from the 2014 Camerdata Business Registry.

12. This database was filtered applying the National Economic Activities Classification (CNAE) codes that the Ministry of Economy and Competitiveness establishes as a guarantee of innovation. The CNAE code groups employed were as follows: High-tech manufacturing (21.26 and 30.3), Mid-high tech manufacturing (20, 27 to 29,30,30.1, 30.3, and 32.5), Hightech or end-to-end services (59 to 63 and 72), ICT manufacturing, IC'T services and Telecommunications. Sample, Maintenance Architecture, 133.

13. This study used the definition of "startup" provided by Fúndeu: a foundation promoted by Agencia Efe, sponsored by BBVA and assessed by the RAE and whose goal is the proper usage of the Spanish language in the media. (Link). (Whats is a startup, Forbes) or its acquisition (Link ACCIO Startup Catalonia).

14. See D.J. Jackson, "What is an Innovation Ecosystem?" National Science Foundation, Arlington, VA (2011) 